\title{
Transmission of SARS-CoV-2 from Human to Domestic Ferret
}

\author{
Jožko Račnik, Ana Kočevar, Brigita Slavec, Miša Korva, Katarina Resman Rus, Samo Zakotnik, \\ Tomaž Mark Zorec, Mario Poljak, Milan Matko, Olga Zorman Rojs, Tatjana Avšič Županc
}

We report a case of natural infection with severe acute respiratory syndrome coronavirus 2 transmitted from an owner to a pet ferret in the same household in Slovenia. The ferret had onset of gastroenteritis with severe dehydration. Whole-genome sequencing of the viruses isolated from the owner and ferret revealed a 2-nt difference.

$\mathrm{N}$ Tatural infections with severe acute respiratory syndrome coronavirus 2 (SARS-CoV-2) in domestic animals living in infected households have been reported (1). Because of their increased popularity as a pet (2), domestic ferrets (Mustela putorius furo) pose a high risk for transmitting anthropozoonotic infections. A recent study in Spain showed that natural SARS-CoV-2 infections can occur in ferrets kept as working animals for rabbit hunting, especially if a high viral circulation is present in the human population (3). Further, ferrets are common laboratory animal models and, at least in experimental conditions, have been shown to be highly susceptible to SARS$\mathrm{CoV}-2$ infection and likely to transmit the virus to other ferrets without apparent clinical signs (4).

\section{The Study}

On November 20, 2020, a 5-year-old neutered male domestic ferret had signs of acute gastroenteritis, including apathy, anorexia, vomiting, and profuse mucous diarrhea. Another ferret in the same household appeared healthy. Because the ferret's condition did not improve, the owner took it to a veterinary hospital for clinical examination on November 23. The ferret was lethargic and, on the basis of skin turgor, was

Author affiliations: University of Ljubljana Faculty of Veterinary Medicine, Ljubljana, Slovenia (J. Račnik, B. Slavec,

O. Zorman Rojs); Toplica Veterinary Hospital, Topolšica,

Slovenia (A. Kočevar, M. Matko); University of Ljubljana Faculty of Medicine, Ljubljana (M. Korva, K. Resman Rus, S. Zakotnik,

T.M. Zorec, M. Poljak, T. Avšič Županc)

DOI: https://doi.org/10.3201/eid2709.210774
$>5 \%$ dehydrated with low body temperature $\left(36.4^{\circ} \mathrm{C}\right.$, reference range $\left.37.8-40^{\circ} \mathrm{C}\right)$ and slow heart rate $(180$ beats/min, reference $200-400$ beats/min). The body condition of the ferret was good, with a bodyweight of $1.3 \mathrm{~kg}$. Several hematology and serum biochemistry results were elevated: red blood cell count (12.36 $\times 10^{6} / \mu \mathrm{L}$, reference 7.01-9.65 $\left.\times 10^{6} / \mu \mathrm{L}\right)$, hemoglobin concentration $(21.2 \mathrm{~g} / \mathrm{dL}$, reference $12.2-16.5 \mathrm{~g} / \mathrm{dL})$, and hematocrit $(0.57 \%$, reference $0.36 \%-0.48 \%)$; blood urea nitrogen (>129.94 mg/dL, reference 18-32 mg/ $\mathrm{dL})$, hyperproteinemia $(8.5 \mathrm{~g} / \mathrm{dL}$, reference $4.5-6.2 \mathrm{~g} /$ $\mathrm{dL})$, hyperglobulinemia $(4.4 \mathrm{~g} / \mathrm{dL}$, reference $2.8-3.6$ $\mathrm{g} / \mathrm{dL})$, and borderline hyperalbuminemia (4.0 g/ $\mathrm{dL}$, reference $2.5-4.0 \mathrm{~g} / \mathrm{dL}$ ) were consistent with dehydration and possible infection. The results of all other hematologic and biochemical values were within reference ranges. Whole-body radiographs (Appendix Figure, https://wwwnc.cdc.gov/EID/ article/27/9/21-0774-App1.pdf) showed splenomegaly and gas accumulation in intestinal loops. Interstitial and alveolar patterns of cranial lung lobes were present, indicating possible lobar pneumonia. The ferret was hospitalized and initially given fluid therapy, amoxicillin, esomeprazole, maropitant, and dexamethasone. Three days later, the clinical status of the ferret improved, hematologic and biochemical values normalized, and the ferret was scheduled for discharge. However, on the same day, the owner informed the veterinary hospital of having positive results for SARS-CoV-2 RNA tested on November 24, after 9 days of malaise. Additional rectal and oropharyngeal swab specimens and blood samples were taken from the ferret for further diagnostic procedures, and the ferret was discharged from the hospital and put into isolation at the owner's home. Samples were not taken from the other pet ferret at the residence, but the rest of household members tested negative for SARS-CoV-2 RNA on November 25.

We tested the ferret's samples for SARSCoV-2 RNA (Appendix) and ferret-specific enteric 
coronavirus (FERCV) (5) by real-time reverse transcription PCR; influenza A and B viruses (6) by reverse transcription PCR; and herpesviruses (7), adenoviruses (8), and circoviruses (9) and by PCR. The only positive result was the detection of SARS-CoV-2 RNA in the rectal and oropharyngeal swab specimens. In the oropharyngeal swab specimen, all 3 targeted genes (envelope, cycle threshold $\left[\mathrm{C}_{\mathrm{t}}\right]$ 27.7; RNA dependent RNA polymerase, $C_{t} 28.5$; and nucleocapsid, $C_{t} 32.1$ ) were detected, and in the rectal swab specimen only envelope gene $\left(C_{t} 35.6\right)$ was detected, a finding probably attributable to a lower virus concentration. To compare the SARS-CoV-2 detected in the owner and the ferret, we conducted whole-genome sequencing on Illumina MiSeq (Illumina, https://www.illumina.com) on the basis of the ARTIC protocol (https://artic.network/ncov-2019/ncov2019-bioinformatics-sop.html). The complete genome sequences were deposited in the GISAID database (https:/ / www.gisaid.org; accession nos. EPI_ISL_1490186 and EPI_ISL_1490187). According to the pangolin nomenclature, the sequences belonged to the B.1.258 Pango lineage, which was on the rise in Slovenia in November 2020 (Figure 1). The comparison of both sequences showed $\approx 100 \%$ identity, differing by 2 nucleotides (position/owner/ferret: 2,097/G/T; 22,832/C/A).

We also confirmed the SARS-CoV-2 infection in the ferret on the basis of SARS-CoV-2 IgG seroconversion and development of neutralizing antibodies. We tested the ferret's acute and convalescent serum samples with an in-house immunofluorescent assay (Appendix). The first serum sample obtained on day 6 after disease onset tested negative; however, seroconversion was observed on day 19, when the IgG titer was 1:1,024 (Figure 2, panels A, B). In addition, we detected a high neutralizing antibody titer of 1:320 in the second serum sample (Figure 2, panel C).

\section{Conclusions}

SARS-CoV-2 originated in animals, jumped into humans, and is now easily transmitted among humans. In addition to spreading from animals to humans, the virus can be transmitted back into animals, as observed in farmed mink (Neovison vison) (10). Most experimentally infected ferrets do not exhibit clinical signs or have only mild fever, lethargy, loss of appetite, and occasional cough $(4,11)$. Also, among working ferrets naturally infected with SARS-CoV-2 in Spain, no signs of illness were reported (3).

In our study, the infected ferret had onset of severe disease with gastroenteritis, pneumonia, and dehydration and required aggressive fluid therapy and supportive care with antibiotics, antacids, antiemetics, and parenteral dexamethasone. The ferret responded to the therapy promptly and fully recovered in 3 days. Acute epizootic catarrhal enteritis caused

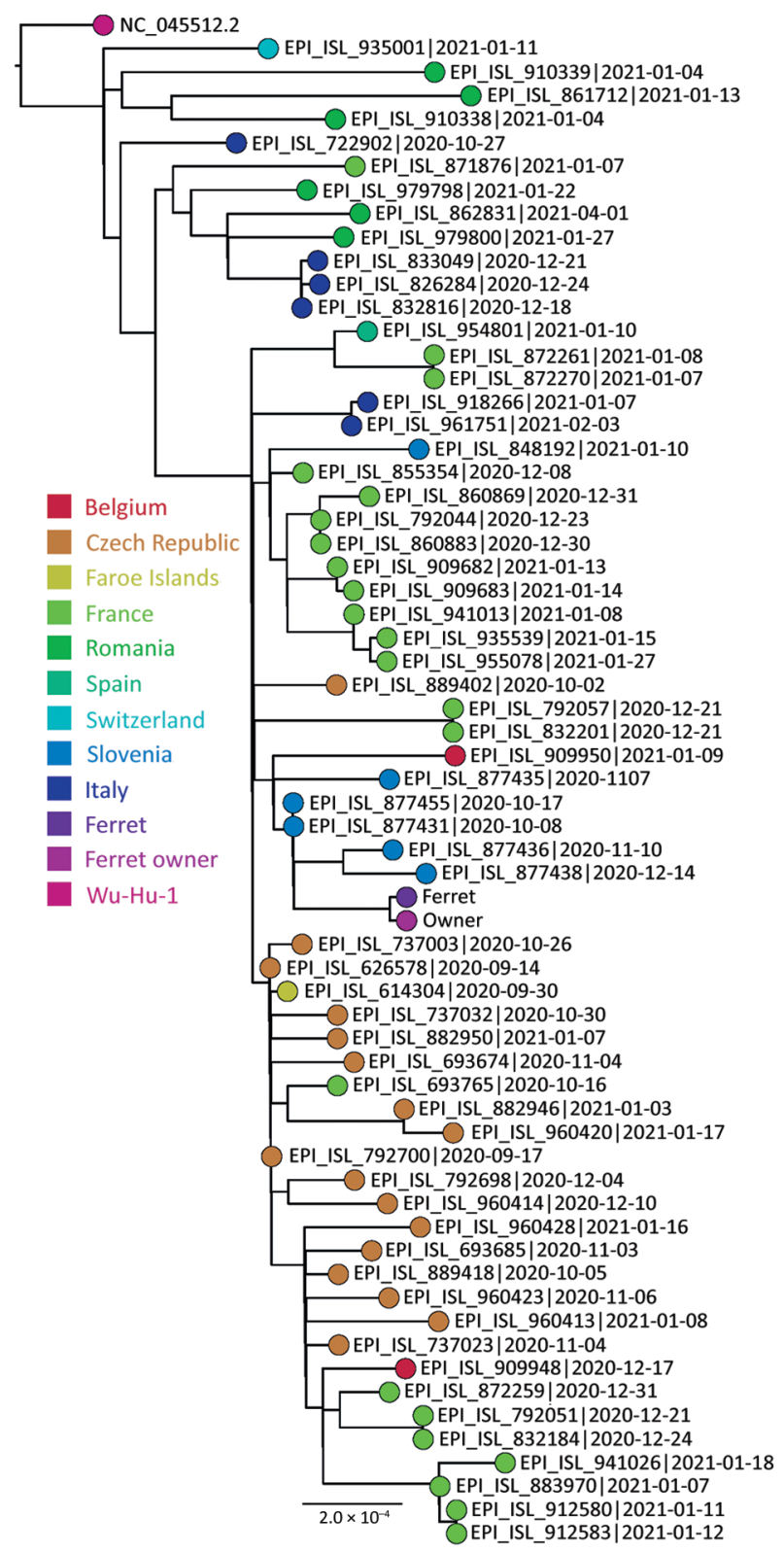

Figure 1. Phylogenetic sequence context consisted of high-quality complete severe acute respiratory syndrome coronavirus 2 genome sequences from a domestic ferret, Slovenia, corresponding to Pango lineage B.1.258. The context sequences were downloaded from GISAID (https://www.gisaid.org) and subsampled to 62 sequences and National Center for Biotechnology Information reference sequence NC_045512.2. The phylogenetic reconstruction using a general time-reversible plus F plus R4 substitution model was built in Figtree (Evomics, http://evomics.org) with 1,000 bootstrap replicates. The reference sequence was used as an outgroup to root the phylogenetic tree. GISAID accession numbers and isolation dates are provided. Scale bar indicates substitutions per site. 

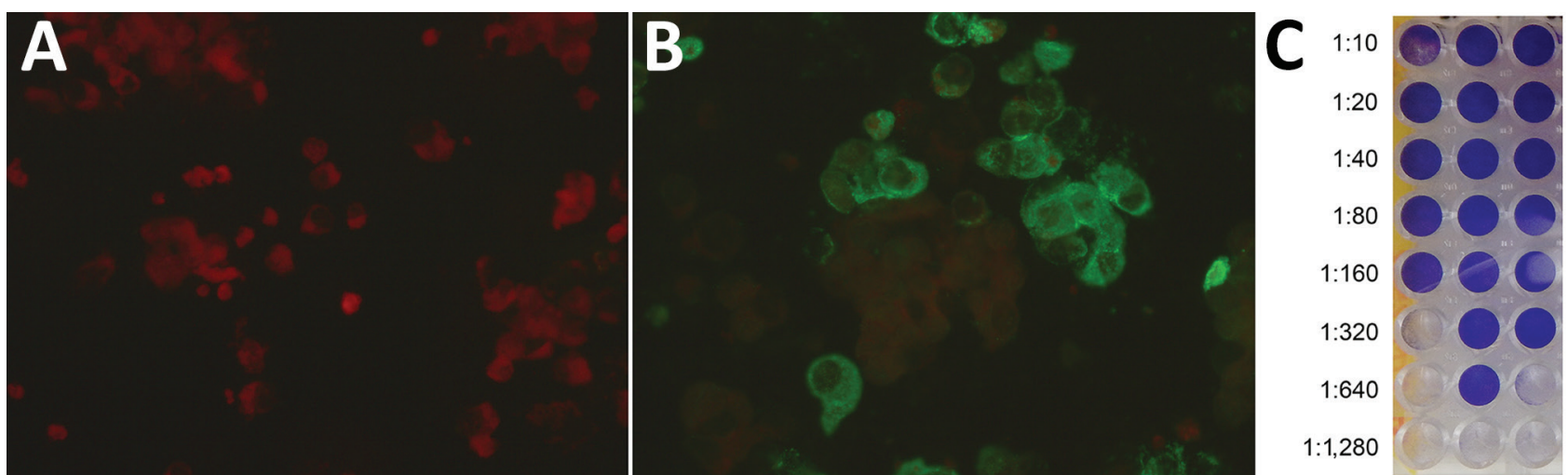

Figure 2. Serological response to infection with severe acute respiratory syndrome coronavirus 2 in a domestic ferret, Slovenia. Immunofluorescent tests showed a negative result in the ferret's acute serum sample obtained on day 6 after disease onset (A) and a positive reaction at titer 1:64 in the ferret's convalescent serum sample obtained on day 19 (B). The neutralization test (C) showed the highest dilution of the ferret's convalescent serum sample that inhibited a cytopathic effect in $\geq 2$ of 3 wells to be $1: 320$.

by FERCV was one of the plausible differential diagnoses in the initial treatment plan for the ferret. For this reason, dexamethasone was used parenterally because additional treatment with a short course of steroids might speed the recovery and reduce future problems of malabsorption attributable to villi destruction caused by fulminate FERCV infection (12). In humans, the effective drugs against coronavirus disease are poorly defined, yet dexamethasone in combination with supportive therapy is frequently used (13). However, the risk for unnecessary use and adverse effects must be considered before treatment attempts with corticosteroids.

We assume that SARS-CoV-2 likely spread from the infected owner to the ferret living in the same household. Symptoms appeared in the owner 4 days before the ferret became ill. All other household members tested negative for SARS-COV-2 RNA, ruling out asymptomatic infected persons in the family. Another close contact ferret in the same household appeared healthy. Likewise, no disease among staff or animals at the veterinary hospital was reported during or after the hospitalization of the ferret. Nevertheless, ferrets as laboratory models were shown to shed SARS-CoV-2 up to 8 days postinfection in nasal swab, saliva, urine, and fecal samples. Ferrets can effectively transmit the infection to other animals (14) or possibly humans, thus highlighting the importance of recognizing the infection in pets early to prevent spread to other animals or humans in the same household or elsewhere (15).

In the mink farm outbreak in Denmark, SARSCoV-2 transmission was shown to spill over from minks to humans accumulating mutations that are resistant to neutralizing antibodies or vaccines along the way (10). In our study, whole-genome sequencing of the virus detected in the owner and ferret revealed only a 2-nt difference, and neither of those was present in the spike protein gene. Nonetheless, retaining the One Health approach is crucial for early detection and monitoring of emerging zoonoses in humans.

\section{Acknowledgments}

We thank all members of the COVID-19 Next

Generation Sequencing team for great technical assistance in sequencing SARS-CoV-2 genomes and the staff at the Toplica Veterinary Hospital for caring for the ferret. We thank the staff at the Institute for Poultry, Birds, Small Mammals, and Reptiles for their assistance in performing PCR assays. Thanks to the owners of the ferret for their kind support and for allowing publication of this report.

This work was supported by the Slovenian Research Agency (grant nos. P3-0083 and V3-2034 at the Institute of Microbiology and Immunology, Faculty of Medicine, University of Ljubljana and P4-0092 at the Faculty of Veterinary Medicine, University of Ljubljana) and by the European Virus Archive Global project, which received funding from the European Union's Horizon 2020 research and innovation program under grant no. 871029 .

\section{About the Author}

Dr. Račnik is an associate professor at the Faculty of Veterinary Medicine, University of Ljubljana, Slovenia, and diplomate of the European College of Zoological Medicine, wildlife population health specialty. His primary research interests include clinical veterinary medicine and emerging diseases of exotic pets and wild birds.

\section{References}

1. Patterson EI, Elia G, Grassi A, Giordano A, Desario C, Medardo M, et al. Evidence of exposure to SARS-CoV-2 
in cats and dogs from households in Italy. Nat Commun. 2020;11:6231. https:// doi.org/10.1038/s41467-020-20097-0

2. Bixler H, Ellis C. Ferret care and husbandry. Vet Clin North Am Exot Anim Pract. 2004;7:227-55, v. https:// doi.org/ 10.1016/j.cvex.2004.02.002

3. Gortázar C, Barroso-Arévalo S, Ferreras-Colino E, Isla J, de la Fuente G, Rivera B, et al. Natural SARS-CoV-2 infection in kept ferrets, Spain. Emerg Infect Dis. 2021;27(7):1994-1996. https://doi.org/10.3201/eid2707.210096

4. Shi J, Wen Z, Zhong G, Yang H, Wang C, Huang B, et al. Susceptibility of ferrets, cats, dogs, and other domesticated animals to SARS-coronavirus 2. Science. 2020;368:1016-20. https://doi.org/10.1126/science.abb7015

5. Muradrasoli S, Mohamed N, Hornyák A, Fohlman J, Olsen B, Belák S, et al. Broadly targeted multiprobe QPCR for detection of coronaviruses: coronavirus is common among mallard ducks (Anas platyrhynchos). J Virol Methods. 2009;159:277-87. https://doi.org/10.1016/j.jviromet. 2009.04.022

6. Boonsuk P, Payungporn S, Chieochansin T, Samransamruajkit R, Amonsin A, Songserm T, et al. Detection of influenza virus types A and B and type A subtypes (H1, H3, and $\mathrm{H} 5)$ by multiplex polymerase chain reaction. Tohoku J Exp Med. 2008;215:247-55. https://doi.org/10.1620/tjem.215.247

7. VanDevanter DR, Warrener P, Bennett L, Schultz ER, Coulter S, Garber RL, et al. Detection and analysis of diverse herpesviral species by consensus primer PCR. J Clin Microbiol. 1996;34:1666-71. https:/ /doi.org/10.1128/ jcm.34.7.1666-1671.1996

8. Wellehan JF, Johnson AJ, Harrach B, Benkö M, Pessier AP, Johnson CM, et al. Detection and analysis of six lizard adenoviruses by consensus primer PCR provides further evidence of a reptilian origin for the atadenoviruses. J Virol. 2004;78:13366-9. https://doi.org/10.1128/ JVI.78.23.13366-13369.2004

9. Halami MY, Nieper H, Müller H, Johne R. Detection of a novel circovirus in mute swans (Cygnus olor) by using nested broad-spectrum PCR. Virus Res. 2008;132:208-12. https://doi.org/10.1016/j.virusres.2007.11.001

10. Koopmans M. SARS-CoV-2 and the human-animal interface: outbreaks on mink farms. Lancet Infect Dis. 2021;21:18-9. https://doi.org/10.1016/S1473-3099(20)30912-9

11. Abdel-Moneim AS, Abdelwhab EM. Evidence for SARS-CoV-2 infection in animal hosts. Pathogens. 2020;9:529. https:/ / doi.org/10.3390/pathogens9070529

12. Perpiñán D, Johnson Delaney CA. Disorders of the digestive system and liver. In: Johnson Delaney CA, editor. Ferret medicine and surgery. Boca Raton (FL): CRC Press; 2017. p. 159-90.

13. Ledford H. Coronavirus breakthrough: dexamethasone is first drug shown to save lives. Nature. 2020;582:469. https:/ / doi.org/10.1038/d41586-020-01824-5

14. Kim Y-I, Kim S-G, Kim S-M, Kim E-H, Park S-J, Yu K-M, et al. Infection and rapid transmission of SARS-CoV-2 in ferrets. Cell Host Microbe. 2020;27:704-709.e2. https:/ / doi.org/ 10.1016/j.chom.2020.03.023

15. United Kingdom Animal and Plant Health Agency. Preventative measures regarding SARS-CoV-2 and ferrets in the UK. 2020 [cited 2021 May 8]. http:/ / apha.defra.gov.uk/ documents/guidance-sars-cov-2-ferrets.pdf

Address for correspondence: Jožko Račnik, Institute for Poultry, Birds, Small Mammals, and Reptiles, Faculty of Veterinary Medicine, University of Ljubljana, Gerbičeva 60, 1000 Ljubljana, Slovenia; email: josko.racnik@vf.uni-lj.si

\section{EID Podcast Developing Biological Reference Materials to Prepare for Epidemics}

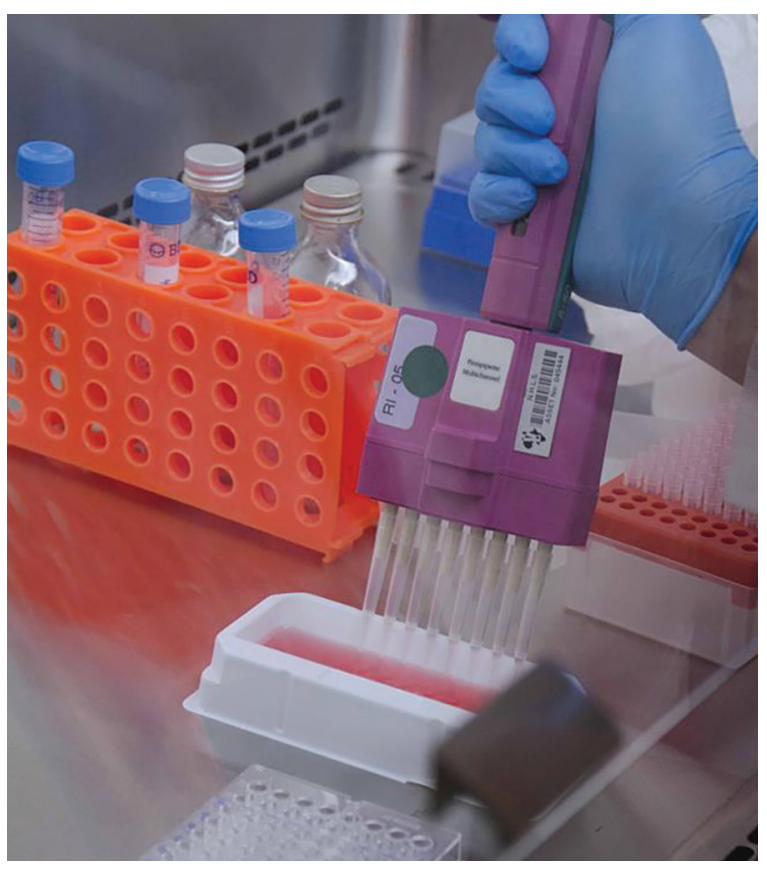

Having standard biological reference materials, such as antigens and antibodies, is crucial for developing comparable research across international institutions. However, the process of developing a standard can be long and difficult.

In this EID podcast, Dr. Tommy Rampling, a clinician and academic fellow at the Hospital for Tropical Diseases and University College in London, explains the intricacies behind the development and distribution of biological reference materials.

\section{Visit our website to listen: https: / /go.usa.gov/xyfJX EMERGING INFECTIOUS DISEASES}

